\title{
Anodische Oxidation von Nickelfeinstein-Suspensionen in konzentrierten NaCl-haltigen Lösungen
}

\author{
Von Chikabumi Yamauchi*, Takeo Oki* und Roland Kammel**
}

\begin{abstract}
Zur Verbesserung der Stromausbeute bei der anodischen Oxidation von Nickelfeinstein-Suspensionen und um näheren Aufschluß über den hierbei stattfindenden Umsetzungsmechanismus zu gewinnen, wurden Suspensionselektrolyse-Versuche und Elektrolyt-Titrationen in Elektrolyten mit $\mathrm{NaCl}$ Gehalten von $0.34 \mathrm{kmol} / \mathrm{m}^{3}$ und $3.0 \mathrm{kmol} / \mathrm{m}^{3}$ bei $363 \mathrm{~K}$ durchgeführt. Die Nickelfeinstein-Suspensionen werden anodisch in drei Stufen, $\mathrm{Ni}^{0}=\mathrm{Ni}^{2+}+2 \mathrm{e}, \mathrm{Ni}_{3} \mathrm{~S}_{2}=\mathrm{Ni}^{2+}+2 \mathrm{NiS}+2 \mathrm{e}$ und $\mathrm{NiS}=\mathrm{Ni}^{2+}+\mathrm{S}^{0}+2 \mathrm{e}$, durch die anodisch gebildeten Chlor oder Chlorverbindungen sowie Sauerstoff oxidiert. Die Versuchsergebnisse zeigen, daß abhängig von der Zusammensetzung des anodisch gebildeten $\mathrm{Cl}_{2} / \mathrm{O}_{2}$-Gasgemisches die anodische Stromausbeute von ca. $83 \%$ auf $96 \%$ erhört werden kann.
\end{abstract}

(Eingegangen am 5. Februar 1986)

Keywords: suspension electrolysis, nickel matte, anodic current efficiency, anodic oxidation, sodium chloride solution

\section{Einleitung}

Frühere Untersuchungen ${ }^{(1)(2)}$ über die direkte anodische Oxidation von NickelfeinsteinSuspensionen der Zusammensetzung 30.8\% metallisches Nickel $\left(\mathrm{Ni}^{0}\right), 58.8 \% \quad \mathrm{Ni}_{3} \mathrm{~S}_{2}$ und 6.1\% $\mathrm{Cu}_{2} \mathrm{~S}$ in schwefelsaurer Lösung mit 0.68 $\mathrm{kmol} / \mathrm{m}^{3} \mathrm{Ni}^{2+}$ als $\mathrm{NiSO}_{4}, 0.34 \mathrm{kmol} / \mathrm{m}^{3} \mathrm{NaCl}$, $0.32 \mathrm{kmol} / \mathrm{m}^{3} \quad \mathrm{H}_{3} \mathrm{BO}_{3}$ und $0.01 \mathrm{~kg} / \mathrm{m}^{3}$ Leim zeigten, daß die anodische Oxidation in folgenden drei Stufen,

$$
\begin{aligned}
\mathrm{Ni}^{0} & =\mathrm{Ni}^{2+}+2 \mathrm{e}, \\
\mathrm{Ni}_{3} \mathrm{~S}_{2} & =\mathrm{Ni}^{2+}+2 \mathrm{NiS}+2 \mathrm{e}
\end{aligned}
$$

und

$$
\mathrm{NiS}=\mathrm{Ni}^{2+}+\mathrm{S}^{0}+2 \mathrm{e},
$$

beim jeweiligen Suspensionspotential (Pt/ Kalomel-Bezugselektrode) von $-0.31--0.38$ $\mathrm{V},-0.20--0.22 \mathrm{~V}$ bzw. rd. $+0.35 \mathrm{~V}$ erfolgt. Hierbei beträgt bei pH-Werten des Anolyten um $\mathrm{pH}=1$ die anodische Stromausbeute einschließlich der chemischen Auflösung des

* Department of Iron \& Steel Engineering, Faculty of Engineering, Nagoya University, Furo-cho, Chikusaku, Nagoya 464, Japan.

** Institut für Metallurgie, Metallhüttenkunde, Technische Universität Berlin, Straße des 17. Juni 135, 1000 Berlin 12, West Germany. metallischen Nickels $\left(\mathrm{Ni}^{0}+2 \mathrm{H}^{+}=\mathrm{Ni}^{2+}+\mathrm{H}_{2}\right)$ höchstens $90 \%$. Bei pH-Werten von 4 bis 5 werden infolge einer geringeren chemischen Auflösung von $\mathrm{Ni}^{0}$ nur anodische Stromausbeutewerte von rd. $80 \%$ erreicht.

Um die anodische Stromausbeute weiter $\mathrm{zu}$ verbessern, wurde bei den ${ }_{\circ}$ nachfolgenden Untersuchungen der $\mathrm{NaCl}-\mathrm{Gehalt}$ von 0.34 $\mathrm{kmol} / \mathrm{m}^{3}$ auf $3.0 \mathrm{kmol} / \mathrm{m}^{3}$ erhöht und die Elektrolyseversuche sowohl mit einer Kleinversuchszelle als auch einer konvexen Zelle ${ }^{(1)}$ durchgeführt.

\section{Versuchsanordnung und -durchführung}

Als Ausgangsmaterial diente Nickelfeinstein der Zusammensetzung $72.94 \% \mathrm{Ni}, 0.96 \% \mathrm{Co}$, $4.86 \% \mathrm{Cu}, 0.19 \% \mathrm{Fe}$ und $16.92 \% \mathrm{~S}$, der den oben erwähnten Bindungsformen entspricht. Der Feinstein mit einer Korngröße von 0.03$0.07 \mathrm{~mm}$ entstammt der gleichen Musterprobe, die bei den früheren Untersuchungen ${ }^{(1)(2)}$ eingesetzt wurde. Gegenüber den früheren Untersuchungen $^{(1)(2)}$ enthält der Elektrolyt neben dem von $0.34 \mathrm{kmol} / \mathrm{m}^{3}$ auf $3.0 \mathrm{kmol} / \mathrm{m}^{3}$ erhöhten $\mathrm{NaCl}-G$ hehalt $0.68 \mathrm{kmol} / \mathrm{m}^{3} \mathrm{Ni}^{2+}$ als $\mathrm{NiSO}_{4}, 0.32 \mathrm{kmol} / \mathrm{m}^{3} \mathrm{H}_{3} \mathrm{BO}_{3}$ und $0.01 \mathrm{~kg} / \mathrm{m}^{3}$ Leim.

Die in Abb. 1 schematisch dargestellte Kleinversuchszelle besteht aus zwei durch eine Salzbrücke verbundenen Becherglässern 


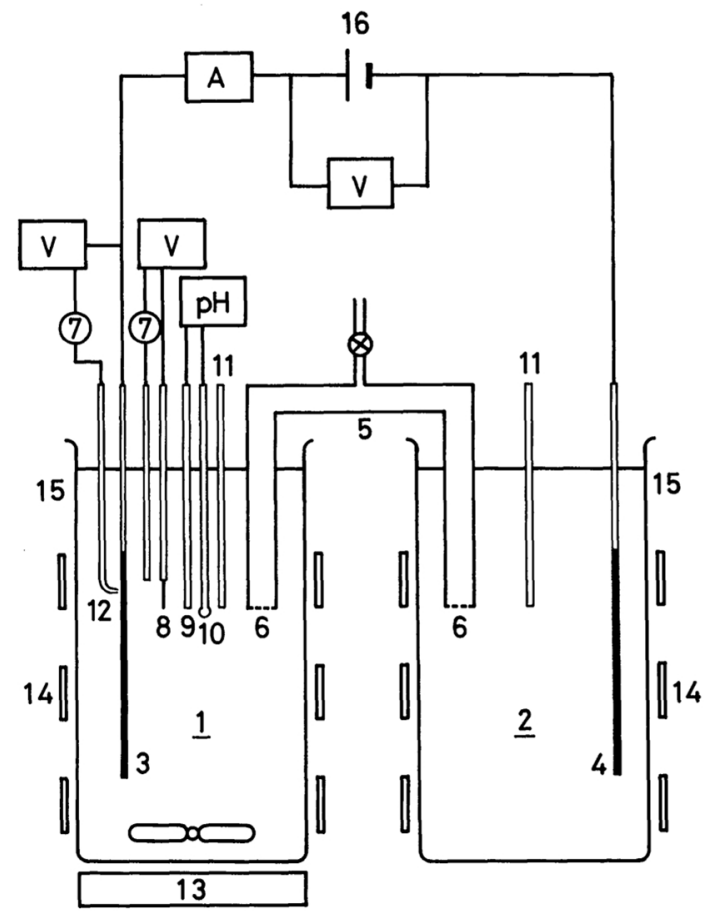

Abb. 1 Schematische Darstellung der Kleinversuchszelle

$\begin{aligned} \frac{1}{2} & \text { Anodenraum } & 9 & \text { Silber-Silberchlorid- } \\ \frac{2}{3} & \text { Platinodenraum } & & \text { Elektrode } \\ 4 & \text { Vernickelte Kupfer- } & 10 & \text { Glaselektrode } \\ & \text { Kathode } & 12 & \text { Thermometer } \\ 5 & \text { Salzbrücke } & 13 & \text { Luggin-Kapillare } \\ 6 & \text { Kunstgewebe } & 14 & \text { Erhitzer } \\ 7 & \text { Kalomel- } & 15 & \text { Becherglas }\left(0.5 \mathrm{dm}^{3}\right) \\ & \text { Bezugselektrode } & 16 & \text { Galvanostat } \\ 8 & \text { Platin-Elektrode } & & \end{aligned}$

von $0.5 \mathrm{dm}^{3}$ Inhalt, die als Anoden- und Kathodenraum dienen. Im Anodenraum(1) sind eine spiralförmige Platin-Anode(3) mit einem Durchmesser von $1.2 \mathrm{~mm}$ und der Länge von $100 \mathrm{~mm}$, ein Erhitzer(14), ein Thermometer(11) sowie Elektroden(7 bis 10) für Potential-und pH-Messungen der Anolyt-Suspension und für Anodenpotentialmessung untergebracht. Der Kathodenraum(2) enthält eine vernickelte Kupfer-Platte(4) als Kathode und ein Thermometer(11). Die beiden Enden der Salzbrücke(5) sind mit Kunstgewebe(6) bedeckt, um ein Vermischung zwischen dem Anolyten und dem Katholyten zu vermeiden. Bei jedem Versuch wurden $15 \mathrm{~g}$ Nickelfeinsteinpulver in den Anodenraum eingesetzt und mit einem Magnet-Rührer(13) suspendiert. Die
Temperatur des Anolyten wurde auf $363 \pm 1 \mathrm{~K}$ konstant gehalten. Nach Schluß der Versuche wurde der Anolyt filtriert und der Rückstand mit destilliertem Wasser und Alkohol gewaschen, bei Raumtemperatur getrocknet und gewogen. Die Bestimmung des Elementalschwefels im Rückstand erfolgte durch $\mathrm{CS}_{2}$-Extraktion. Der Rückstand wurde nach $\mathrm{CS}_{2}$-Extraktion auf $\mathrm{Ni}, \mathrm{Co}, \mathrm{Fe}, \mathrm{Cu}$ und $\mathrm{S}$ analysiert.

Der Versuchsaufbau der konvexen Zelle und die Versuchsdurchführung sind der frühen Untersuchung ${ }^{(1)}$ übernommen worden.

\section{III. Über die Bildung von Oxidations- mittel an der Anode}

Im NaCl-haltigen Anolyten werden an der Platin-Anode bei hoher Sauerstoff-Überspannung hauptsächlich $\mathrm{Cl}^{-}$-Ionen entladen und es entwickelt sich $\mathrm{Cl}_{2}$-Gas (Gl. (4)),

$$
2 \mathrm{Cl}^{-}=\mathrm{Cl}_{2}(\mathrm{~g})+2 \mathrm{e} \text {, }
$$

aus dem entsprechend nachfolgenden Gleichungen (Gln. (5) bis (8)) folgende weitere Oxidationsmittel gebildet werden können:

$$
\begin{gathered}
\mathrm{Cl}_{2}(\mathrm{~g})=\mathrm{Cl}_{2}(\mathrm{aq}) . \\
\mathrm{Cl}_{2}(\mathrm{aq})+\mathrm{H}_{2} \mathrm{O}=\mathrm{HClO}(\mathrm{aq})+\mathrm{H}^{+}+\mathrm{Cl}^{-} . \\
\mathrm{HClO}(\mathrm{aq})=\mathrm{ClO}^{-}+\mathrm{H}^{+} . \\
2 \mathrm{HClO}(\mathrm{aq})+\mathrm{ClO}^{-}=\mathrm{ClO}_{3}^{-}+2 \mathrm{H}^{+}+2 \mathrm{Cl}^{-} .
\end{gathered}
$$

Darin bedeutet (aq) den in der wässerigen Lösung gelösten molekularen Anteil. Von diesen vier Reaktionen laufen die chemische Auflösung von $\mathrm{Cl}_{2}(\mathrm{~g})$ (Gl. (5)), die Disproportionierung von $\mathrm{Cl}_{2}$ (aq) (Gl. (6)) und die Dissoziation von $\mathrm{HClO}(\mathrm{aq})$ (Gl. (7)) sehr schnell $\mathrm{ab}^{(3)(4)}$. Für die Bildung von $\mathrm{ClO}_{3}^{-}$werden im Schrifttum ${ }^{(5)(6)}$ zwei Reaktionswege erwähnt, nämlich, eine Reaktion dritter Ordnung mit 2 Molen $\mathrm{HClO}(\mathrm{aq})$ and $1 \mathrm{Mol} \mathrm{ClO}^{-}$ $(\mathrm{Gl} .(8))^{(5)}$ und eine Reaktion zweiter Ordnung mit nur 2 Molen HClO(aq) wie Gln. (9) und (10) zeigen,

$$
2 \mathrm{HClO}(\mathrm{aq})=\mathrm{ClO}_{2}^{-}+2 \mathrm{H}^{+}+\mathrm{Cl}^{-}
$$

und

$$
\mathrm{HClO}(\mathrm{aq})+\mathrm{ClO}_{2}^{-}=\mathrm{ClO}_{3}^{-}+\mathrm{H}^{+}+\mathrm{Cl}^{-} \text {, }
$$

wobei Gl. (9) der geschwindigkeitsbestim- 
Tab. 1 Gleichgewichtskonstanten für $\mathrm{Cl}_{2}(\mathrm{~g})-\mathrm{Cl}_{2}(\mathrm{aq})-\mathrm{HClO}(\mathrm{aq})-\mathrm{ClO}^{-}-$System bei $363 \mathrm{~K}$.

\begin{tabular}{|c|c|c|c|}
\hline Reaktionen & Definition von $K$ & $p K$ & $K$ \\
\hline $\mathrm{Cl}_{2}(\mathrm{~g})=\mathrm{Cl}_{2}(\mathrm{aq})$ & $K_{\mathrm{a}}=\frac{a_{\mathrm{Cl}_{2}}(\mathrm{aq})}{P_{\mathrm{Cl}_{2}}(\mathrm{~g})}$ & 2.250 & $5.62 \times 10^{-3}$ \\
\hline $\mathrm{Cl}_{2}(\mathrm{~g})+\mathrm{H}_{2} \mathrm{O}=\mathrm{HClO}(\mathrm{aq})+\mathrm{H}^{+}+\mathrm{Cl}^{-}$ & $K_{\mathrm{b}}=\frac{a_{\mathrm{HClO}(\mathrm{aq})} \cdot a_{\mathrm{H}^{+}} \cdot a_{\mathrm{Cl}^{-}}}{P_{\mathrm{Cl}_{2}(\mathrm{~g})} \cdot a_{\mathrm{H}_{2} \mathrm{O}}}$ & 4.757 & $1.75 \times 10^{-5}$ \\
\hline $\mathrm{HClO}(\mathrm{aq})=\mathrm{H}^{+}+\mathrm{ClO}^{-}$ & $K_{\mathrm{c}}=\frac{a_{\mathrm{H}^{+}} \cdot a_{\mathrm{ClO}^{-}}}{a_{\mathrm{HClO}(\mathrm{aq})}}$ & 6.963 & $1.09 \times 10^{-7}$ \\
\hline $\mathrm{Cl}_{2}(\mathrm{~g})+\mathrm{H}_{2} \mathrm{O}=\mathrm{ClO}^{-}+2 \mathrm{H}^{+}+\mathrm{Cl}^{-}$ & $K_{\mathrm{d}}=\frac{a_{\mathrm{ClO}^{-}} \cdot a_{\mathrm{H}^{+}}^{2} \cdot a_{\mathrm{Cl}^{-}}}{P_{\mathrm{Cl}_{2}(\mathrm{~g})} \cdot a_{\mathrm{H}_{2} \mathrm{O}}}$ & 11.720 & $1.91 \times 10^{-12}$ \\
\hline $\mathrm{Cl}_{2}(\mathrm{aq})+\mathrm{H}_{2} \mathrm{O}=\mathrm{HClO}(\mathrm{aq})+\mathrm{H}^{+}+\mathrm{Cl}^{-}$ & $K_{\mathrm{e}}=\frac{K_{\mathrm{b}}}{K_{\mathrm{a}}}$ & 2.507 & $3.11 \times 10^{-3}$ \\
\hline $\mathrm{Cl}_{2}(\mathrm{aq})+\mathrm{H}_{2} \mathrm{O}=\mathrm{ClO}^{-}+2 \mathrm{H}^{+}+\mathrm{Cl}^{-}$ & $K_{\mathrm{f}}=\frac{K_{\mathrm{d}}}{K_{\mathrm{a}}}$ & 9.469 & $3.40 \times 10^{-10}$ \\
\hline $3 \mathrm{Cl}_{2}(\mathrm{~g})+3 \mathrm{H}_{2} \mathrm{O}=\mathrm{ClO}_{3}^{-}+5 \mathrm{Cl}^{-}+6 \mathrm{H}^{+}$ & $K_{\mathrm{g}}=\frac{a_{\mathrm{ClO}_{3}^{-}} \cdot a_{\mathrm{Cl}^{-}}^{5} \cdot a_{\mathrm{H}^{+}}^{6}}{P_{\mathrm{Cl}_{2}(\mathrm{aq})}^{3} \cdot a_{\mathrm{H}_{2} \mathrm{O}}^{3}}$ & 12.645 & $2.27 \times 10^{-13}$ \\
\hline
\end{tabular}

mende Teilschritt ist ${ }^{(6)}$. Hierbei dominiert der Reaktionsmechanismus von Gl. $(8)^{(7)-(10)}$. Die Reaktionsgeschwindigkeit von Gl. (8) erreicht im $\mathrm{pH}$-Bereich von 6-7 einen Maximalwert, ist aber im Vergleich zu derjenigen von Gln. (5), (6) und (7) relativ langsam, insbesondere im neidrigen $\mathrm{pH}-\mathrm{Bereich}^{(3)(4)}$. Von den in $\mathrm{NaCl}$ Lösungen gelösten Gehalten an $\mathrm{Cl}_{2}(\mathrm{aq})$, $\mathrm{HClO}(\mathrm{aq}), \mathrm{ClO}^{-}$und $\mathrm{ClO}_{3}^{-}$wird die Summe der Konzentrationen von $\mathrm{Cl}_{2}(\mathrm{aq}), \mathrm{HClO}(\mathrm{aq})$ sowie $\mathrm{ClO}^{-}$im Gleichgewicht mit $\mathrm{Cl}_{2}(\mathrm{~g})$ bei $101.325 \mathrm{kPa}$ als sog. "Wirksames Chlor" bezeichnet.

Die Gleichgewichtskonstante(K) der auf wirksames Chlor bezogenen Reaktionen sind von Takahashi ${ }^{(3)}$ als $p K$-Wert $(p K=-\log K)$ für 298, 333, 353 und $373 \mathrm{~K}$ angegeben. Daraus lassen sich für die Versuchstemperatur von $363 \mathrm{~K}$ die in Tab. 1 zusammengestellten $p K$-Werte abschätzen.

Die Aktivitätskoeffizienten von $\mathrm{Cl}^{-}$und vom wirksamen Chlor wurden berechnet nach den Beziehungen $\log y_{\mathrm{Cl}_{2}(\mathrm{aq})}=0.12 C_{\mathrm{NaCl}}$ und $\log y_{\mathrm{HClO}(\mathrm{aq})}=0.07 C_{\mathrm{NaCl}}{ }^{(3)(4)}$, wobei $\mathrm{C}$ die Molarität $\left(\mathrm{mol} / \mathrm{dm}^{3}\right)$ und $y$ der auf die Molarität bezogene Aktivitätskoeffizient ist, sowie unter den Voraussetzungen, daß (1) $y_{\mathrm{Cl}_{2} \text { (aq) }}$ und $y_{\mathrm{HClO}(\mathrm{aq})}$ von der Temperatur unabhängig sind, (2) der Akitivitätskoeffizient des $\mathrm{Cl}^{-}$-Ions dem mittleren Aktivitätskoeffizienten von $\mathrm{NaCl}$
Tab. 2 Aktivitätskoeffizienten von $\mathrm{Cl}^{-}, \mathrm{Cl}_{2}(\mathrm{aq})$, $\mathrm{HClO}(\mathrm{aq}), \mathrm{ClO}^{-}$und $\mathrm{H}^{+}$in $0.34 \mathrm{kmol} / \mathrm{m}^{3}$ und 3.0 $\mathrm{kmol} / \mathrm{m}^{3} \mathrm{NaCl}$-haltigen Lösungen bei $363 \mathrm{~K}$.

\begin{tabular}{l|ccccc}
\hline \hline $\begin{array}{l}C_{\mathrm{NaCl}} \\
\left(\mathrm{kmol} / \mathrm{m}^{3}\right)\end{array}$ & $\mathrm{Cl}^{-}$ & $\mathrm{Cl}_{2}(\mathrm{aq})$ & $\mathrm{HClO}(\mathrm{aq})$ & $\mathrm{ClO}^{-}$ & $\mathrm{H}^{+}$ \\
\hline 0.34 & 0.676 & 1.10 & 1.05 & 0.676 & 1.13 \\
3.0 & 0.766 & 2.29 & 1.63 & 0.766 & 2.82 \\
\hline
\end{tabular}

gleich ist, dessen Werte sowohl für verschiedene NaCl-Konzentrationen als auch verschiedene Temperaturen gemessen worden sind ${ }^{(11)}$, und (3) $y_{\mathrm{ClO}-} y_{\mathrm{Cl}^{-}}$gleich ist. Der Aktivitätskoeffizient von $\mathrm{H}^{+}$kann in konzentrierten NaCl-Lösungen durch $\log y_{\mathrm{H}^{+}}=b C_{\mathrm{NaCl}}$ ausgedrückt werden. Als Werte für $b$ sind im Schrifttum 0.20, 0.18 bzw. 0.17 für 298, 313 bzw. $333 \mathrm{~K}$ angegeben ${ }^{(3)(4)}$. Aus diesen Angaben ist abzuschätzen, daß sich für $363 \mathrm{~K}$ ein Wert von 0.15 für $b$ ergibt und daher log $y_{\mathrm{H}^{+}}=0.15 C_{\mathrm{NaCl}}$ wird. Die in dieser Weise berechneten Aktivitätskoeffizienten für $363 \mathrm{~K}$ sind in Tab. 2 zusammengefaßt.

Aus den obigen Gleichgewichtskonstanten und Aktivitätskoeffizienten können für die pH-Abhängigkeit des wirksamen Chlors im Gleichgewicht mit $\mathrm{Cl}_{2}(\mathrm{~g})$ von $101.325 \mathrm{kPa}$ in $0.34 \mathrm{kmol} / \mathrm{m}^{3}$ und $3.0 \mathrm{kmol} / \mathrm{m}^{3} \mathrm{NaCl}$ Lösungen bei $363 \mathrm{~K}$ die in Tab. 3 wiedergegebenen Beziehungen abgeleitet werden, die 
Tab. 3 pH-Abhängigkeit der Konzentration und der Aktivität vom wirksamen Chlor im Gleichgewicht mit $\mathrm{Cl}_{2}$ (g) von $101.325 \mathrm{kPa}$ in $0.34 \mathrm{kmol} / \mathrm{m}^{3}$ und $3.0 \mathrm{kmol} /$ $\mathrm{m}^{3} \mathrm{NaCl}$-haltigen Lösungen bei $363 \mathrm{~K}$.

\begin{tabular}{ll}
\hline$C_{\mathrm{NaCl}}=0.34 \mathrm{kmol} / \mathrm{m}^{3}$ & $C_{\mathrm{NaCl}}=3.0 \mathrm{kmol} / \mathrm{m}^{3}$ \\
\hline$p a_{\mathrm{Cl}_{2} \text { (aq) }}=2.250$ & $p a_{\mathrm{Cl}_{2} \text { (aq) }}=2.250$ \\
$p a_{\mathrm{HClO}(\mathrm{aq})}=4.118-\mathrm{pH}$ & $p a_{\mathrm{HClO}(\mathrm{aq})}=5.118-\mathrm{pH}$ \\
$p a_{\mathrm{ClO}^{-}}=11.081-2 \mathrm{pH}$ & $p a_{\mathrm{ClO}^{-}}=12.081-2 \mathrm{pH}$ \\
\hline$p C_{\mathrm{Cl}_{2} \text { (aq) }}=2.292$ & $p C_{\mathrm{Cl}_{2} \text { (aq) }}=2.611$ \\
$p C_{\mathrm{HClO}^{(a q)}}=4.140-\mathrm{pH}$ & $p C_{\mathrm{HClO}^{(a q)}}=5.331-\mathrm{pH}$ \\
$p C_{\mathrm{ClO}^{-}}=10.911-2 \mathrm{pH}$ & $p C_{\mathrm{ClO}^{-}}=11.966-2 \mathrm{pH}$ \\
\hline \hline
\end{tabular}
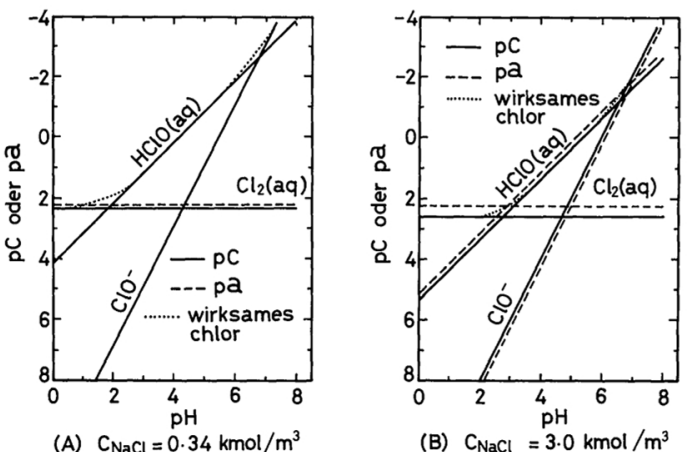

Abb. 2 pH-Abhängigkeit der Konzentration und der Aktivität von $\mathrm{Cl}_{2}(\mathrm{aq}), \mathrm{HClO}(\mathrm{aq})$ und $\mathrm{ClO}^{-}$in 0.34 $\mathrm{kmol} / \mathrm{m}^{3}$ und $3.0 \mathrm{kmol} / \mathrm{m}^{3} \mathrm{NaCl}$-haltigen Lösungen im Gleichgewicht mit $\mathrm{Cl}_{2}(\mathrm{~g})$ von $101.325 \mathrm{kPa}$ bei $363 \mathrm{~K}$

auch in Abb. 2 dargestellt sind. Die Auswertung zeigt, daß der Unterschied zwischen $p a$ and $p C$ von $\mathrm{HClO}(\mathrm{aq})$ und $\mathrm{ClO}^{-}$bei $C_{\mathrm{NaCl}}$ $=0.34 \mathrm{kmol} / \mathrm{m}^{3}$ gering ist und daß unter den Versuchsbedingungen bei $\mathrm{pH}<5$ der Einfluß von $\mathrm{ClO}^{-}$vernachlässigt werden kann. Das wirksame chlor wird ausschließlich von den Gehalten an $\mathrm{Cl}_{2}(\mathrm{aq})$ und/order $\mathrm{HClO}(\mathrm{aq})$ bestimmt $u$. zw. dominiert bei $C_{\mathrm{NaCl}}=0.34$ $\mathrm{kmol} / \mathrm{m}^{3} \mathrm{Cl}_{2}(\mathrm{aq})$ für $\mathrm{pH}<\mathrm{ca} .1 .9, \mathrm{HClO}(\mathrm{aq})$ für $\mathrm{pH}>$ ca. 1.9 und bei $C_{\mathrm{NaCl}}=3.0 \mathrm{kmol} / \mathrm{m}^{3}$ $\mathrm{Cl}_{2}$ (aq) für $\mathrm{pH}<$ ca. 2.7, $\mathrm{HClO}$ (aq) für $\mathrm{pH}>$ ca. 2.7.

\section{IV. $\mathrm{H}_{2} \mathrm{SO}_{4}$ - und HCl-Titration des Elektrolyten}

Um bei den nachfolgenden Suspensionselektrolysen die pH-Änderungen des Anolyten bestimmen zu können, wurde durch Titration von Lösungen mit $\mathrm{NiSO}_{4}, \mathrm{Na}_{2} \mathrm{SO}_{4}, \mathrm{NaCl}$

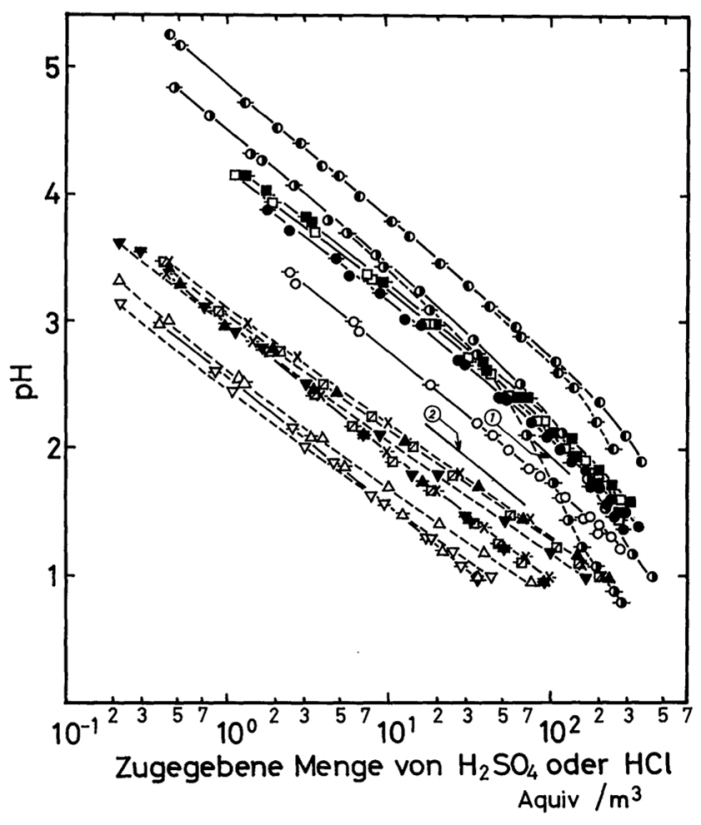

Abb. 3 Titrationskurven der $\mathrm{NiSO}_{4^{-}}, \mathrm{Na}_{2} \mathrm{SO}_{4^{-}}, \mathrm{NaCl}-$ und/order $\mathrm{H}_{3} \mathrm{BO}_{3}$-haltigen Lösungen durch $\mathrm{H}_{2} \mathrm{SO}_{4}$ - und HCl-Lösungen

$\times$ Destilliertes Wasser

- $\mathrm{NaCl}=0.34 \mathrm{kmol} / \mathrm{m}^{3}$

$\triangle \mathrm{NaCl}=3.0 \mathrm{kmol} / \mathrm{m}^{3}$

$\nabla \mathrm{NaCl}=0.34 \mathrm{kmol} / \mathrm{m}^{3}, \mathrm{H}_{3} \mathrm{BO}_{3}=0.32 \mathrm{kmol} / \mathrm{m}^{3}$

$\nabla \mathrm{NaCl}=3.0 \mathrm{kmol} / \mathrm{m}^{3}, \mathrm{H}_{3} \mathrm{BO}_{3}=0.32 \mathrm{kmol} / \mathrm{m}^{3}$

- $\mathrm{NiSO}_{4}=0.68 \mathrm{kmol} / \mathrm{m}^{3}$

$\square \quad \mathrm{NiSO}_{4}=0.68 \mathrm{kmol} / \mathrm{m}^{3}, \mathrm{H}_{3} \mathrm{BO}_{3}=0.32 \mathrm{kmol} / \mathrm{m}^{3}$

$\square \quad \mathrm{H}_{3} \mathrm{BO}_{3}=0.32 \mathrm{kmol} / \mathrm{m}^{3}$

(1) $\mathrm{Na}_{2} \mathrm{SO}_{4}=0.1 \mathrm{kmol} / \mathrm{m}^{3}$

(1) $\mathrm{Na}_{2} \mathrm{SO}_{4}=0.5 \mathrm{kmol} / \mathrm{m}^{3}$

- $\mathrm{NiSO}_{4}=0.68 \mathrm{kmol} / \mathrm{m}^{3}, \mathrm{NaCl}=0.34 \mathrm{kmol} / \mathrm{m}^{3}$, $\mathrm{H}_{3} \mathrm{BO}_{3}=0.32 \mathrm{kmol} / \mathrm{m}^{3}$

. $\mathrm{NiSO}_{4}=0.68 \mathrm{kmol} / \mathrm{m}^{3}, \mathrm{NaCl}=3.0 \mathrm{kmol} / \mathrm{m}^{3}$, $\mathrm{H}_{3} \mathrm{BO}_{3}=0.32 \mathrm{kmol} / \mathrm{m}^{3}$

(1) $\mathrm{NiSO}_{4}=0.68 \mathrm{kmol} / \mathrm{m}^{3}, \mathrm{NaCl}=0.34 \mathrm{kmol} / \mathrm{m}^{3}$ $\mathrm{H}_{3} \mathrm{BO}_{3}=0.32 \mathrm{kmol} / \mathrm{m}^{3}, \mathrm{NiCl}_{2}=0.122 \mathrm{kmol} / \mathrm{m}^{3}$ $\left(C_{\mathrm{Cl}^{-}}=0.584 \mathrm{kmol} / \mathrm{m}^{3}\right)$

(2) $\mathrm{NiSO}_{4}=0.68 \mathrm{kmol} / \mathrm{m}^{3}, \mathrm{NaCl}=3.0 \mathrm{kmol} / \mathrm{m}^{3}$ $\mathrm{H}_{3} \mathrm{BO}_{3}=0.32 \mathrm{kmol} / \mathrm{m}^{3}, \mathrm{NiCl}_{2}=0.67 \mathrm{kmol} / \mathrm{m}^{3}$ $\left(C_{\mathrm{Cl}^{-}}=4.34 \mathrm{kmol} / \mathrm{m}^{3}\right)$

Zeichen mit Bart: HCl-Titration

Zeichen ohne Bart: $\mathrm{H}_{2} \mathrm{SO}_{4}$-Titration

und/order $\mathrm{H}_{3} \mathrm{BO}_{3}$ durch $\mathrm{H}_{2} \mathrm{SO}_{4^{-}}$sowie $\mathrm{HCl}$ Lösung die Abhängigkeit thermodynamischer Voraussetzungen vom pH-Wert ermittelt. Aus den in $\mathrm{Abb} .3$ für Lösungen ohne $\mathrm{SO}_{4}^{2-}$-Ionen dargestellten Ergebnissen geht hervor: daß der Aktivitätskoeffizient der $\mathrm{H}^{+}$-Ionen, $y_{\mathrm{H}^{+}}$, nahezu gleich 1 bei destilliertem Wasser und der Lösung mit $0.32 \mathrm{kmol} / \mathrm{m}^{3} \mathrm{H}_{3} \mathrm{BO}_{3}$ ist; daß 
in NaCl-Lösung der sog. Aussalzeffekt auftritt, insbesondere merklich bei der Konzentration von $3.0 \mathrm{kmol} / \mathrm{m}^{3}$, wobei sich für $y_{\mathrm{H}^{+}}$ein Wert von 3.0 ergibt, der mit dem Wert von 2.82 in Tab. 2 gut übereinstimmt; daß bei der Titration $\mathrm{NaCl}$ - und $\mathrm{H}_{3} \mathrm{BO}_{3}$-haltiger Lösungen mit $\mathrm{H}_{2} \mathrm{SO}_{4}$ sich $y_{\mathrm{H}^{+-}}$Werte scheinbar ergeben, die größer sind als in Lösungen, die nur $\mathrm{NaCl}$ order $\mathrm{H}_{3} \mathrm{BO}_{3}$ enthalten. Diese Abhängingkeit konnte bei der $\mathrm{HCl}$-Titration nicht festgestellt werden. In sulfathaltigen Lösungen wurden demgegenüber die Titrationskurven in einen höheren $\mathrm{pH}$-Bereich verschoben und im $\mathrm{pH}$ Zwischenbereich kann jede Titrationskurve durch eine Gerade mit der Neigung von -1 dargestellt werden. In diesem Bereich stimmen die $\mathrm{HCl}$ - und $\mathrm{H}_{2} \mathrm{SO}_{4}$-Titrationskurven miteinander überein. Aus Abb. 3 ist ferner zu entnehmen, daß im höheren und im neidrigeren $\mathrm{pH}-$ Bereich die Titrationskurven von dieser Gerade abweichen. Diese Abweichung ist im neidrigen $\mathrm{pH}$-Bereich bei der HCl-Titration deutlicher ausgeprägt als bei der $\mathrm{H}_{2} \mathrm{SO}_{4}$-Titration. Im extrem niedrigen $\mathrm{pH}$-Bereich zeigt jede Titrationskurve die Tendenz, sich seiner entsprechenden Titrationskurve für destilliertes Wasser zu nähern. Die Koexistenz von $\mathrm{NaCl}$ ruft den gleichen Aussalzeffekt hervor, wie bei destilliertem Wasser.

\section{Versuchsergebnisse der Suspen- sionselektrolyse und Diskussion}

\section{Kleinversuchszelle}

Abb. 4 zeight beim Zellenstrom von 0.7 A(Vers.-Nr.: K-1, $\left.C_{\mathrm{NaCl}}=0.34 \mathrm{kmol} / \mathrm{m}^{3}\right)$ und $1.5 \mathrm{~A}$ (Vers.-Nr.: $\left.\mathrm{K}-2, \quad C_{\mathrm{NaCl}}=3.0 \mathrm{kmol} / \mathrm{m}^{3}\right)$ den zeitlichen Verlauf des Anodenpotentials, des Suspensionspotentials und des $\mathrm{H}^{+}$Verhältnisses, d. h. des Verhältnisses der jeweiligen $\mathrm{H}^{+}$-Konzentration zur $\mathrm{H}^{+}$-Konzentration am Schluß der Elektrolyse im Anolyten, die vom pH-Meßwert mit Hilfe der Abb. 3 umgerechnet wurden, unter der Voraussetzung, daß der $\mathrm{Cl}^{-}$-Konzentration im Anolyten während der Elektrolyse unverändert ist. Beide Elektrolyse-Versuchsreihen zeigen die drei ausgeprägten Oxidationsstufen (s. Gln. (1), (2) und (3)). Die Ausbeute $\left(\varepsilon_{s}\right)$ der

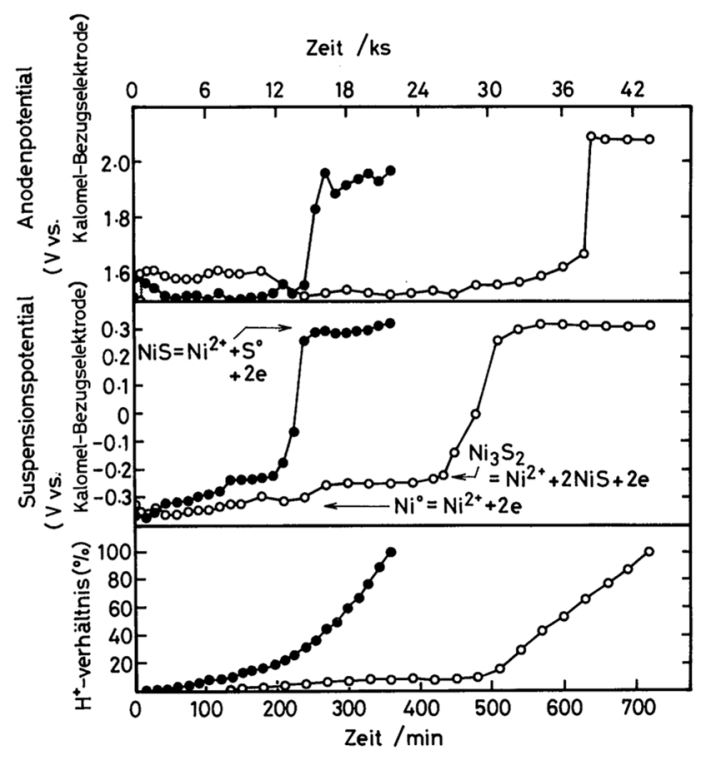

Abb. 4 Zeitlicher Verlauf der Elektrolyse bei der Kleinversuchszelle

K -1 ,

Erläuterung im Text.

Elementalschwefelbildung (s. Schrifttum ${ }^{(1)}$ für die Berechnungsmethode) war ca. $90 \%$ bei $C_{\mathrm{NaCl}}=0.34 \mathrm{kmol} / \mathrm{m}^{3}$ und ca. $103 \%$ bei $C_{\mathrm{NaCl}}=3.0 \mathrm{kmol} / \mathrm{m}^{3}$. Im Elektrolyten mit 3.0 $\mathrm{kmol} / \mathrm{m}^{3} \mathrm{NaCl}$ nimmt das $\mathrm{H}^{+}$-Verhältnis während des Elektrolyseverlaufs zu, während im $0.34 \mathrm{kmol} / \mathrm{m}^{3} \mathrm{NaCl}$-haltigen Elektrolyten erst in der dritten Oxidationsstufe ein rascher und geradliniger Anstieg zu verzeichnen ist.

Aus den in Tab. 4 zusammengestellten Stromausbeute-Werten geht hervor, daß in Lösungen mit $0.34 \mathrm{kmol} / \mathrm{m}^{3} \mathrm{NaCl}$ Stromausbeuten um $93 \%$ und im $3.0 \mathrm{kmol} / \mathrm{m}^{3} \mathrm{NaCl}$ haltigen Elektrolyten Werte bis zu $98 \%$ für die Anodische Oxidation von $\mathrm{Ni}, \mathrm{Co}$ and $\mathrm{Cu}$ $\left(\eta_{\mathrm{Ni}, \mathrm{Co}, \mathrm{Cu}}\right)$ unter der Annahme einer zweiwertigen Auflösung von Kupfer bei $0.34 \mathrm{kmol} / \mathrm{m}^{3}$ und einer einwertigen bei $3.0 \mathrm{kmol} / \mathrm{m}^{3} \mathrm{zu}$ verzeichen sind. Am rechten Ende der Tab. sind die Werte für die $\mathrm{Cl}_{2}-\left(\eta_{\mathrm{Cl}_{2}}\right)$ und $\mathrm{O}_{2^{-}}$ Entwicklung $\left(\eta_{\mathrm{O}_{2}}\right)$ angegeben, die aus Analysenwerten der während des stationären Zustands der Elektrolyse in Elektrolyten ohne Nickelfeinsteinsuspensionen an der Pt-Anode entwickelten Gasen errechnet wurden. Im Falle von $0.34 \mathrm{kmol} / \mathrm{m}^{3}$ ist $\eta_{\mathrm{Ni}, \mathrm{Co}, \mathrm{Cu}}$ viel größer als 
Tab. 4 Stromausbeute für die anodische Oxidation von $\mathrm{Ni}, \mathrm{Co}$ und $\mathrm{Cu}\left(\eta_{\mathrm{Ni}, \mathrm{Co}, \mathrm{Cu}}\right)$ und für $\mathrm{H}^{+}$-Entstehung $\left(\eta_{\mathrm{H}^{+}}\right)$bei der Suspensionselektrolyse sowie diejenige für $\mathrm{Cl}_{2}-\left(\eta_{\mathrm{Cl}_{2}}\right)$ und $\mathrm{O}_{2}$-Entwicklung $\left(\eta_{\mathrm{O}_{2}}\right)$ bei der Elektrolyse des Elektrolyten ohne Nickelfeinstein-Suspensionen.

\begin{tabular}{|c|c|c|c|c|c|c|c|c|c|c|c|c|}
\hline \multirow{5}{*}{$\begin{array}{l}\text { Versuch- } \\
\text { Nr. }\end{array}$} & \multirow{5}{*}{$\begin{array}{c}C_{\mathrm{NaCl}} \\
\left(\mathrm{kmol} / \mathrm{m}^{3}\right)\end{array}$} & \multicolumn{11}{|c|}{ Stromausbeute $(\%)$} \\
\hline & & \multirow{4}{*}{$\eta_{\mathrm{Ni}, \mathrm{Co}, \mathrm{Cu}}$} & \multicolumn{8}{|c|}{ Suspensionselektrolyse } & \multicolumn{2}{|c|}{$\begin{array}{l}\text { Elektrolyse des } \\
\text { Elektrolyten }\end{array}$} \\
\hline & & & \multicolumn{4}{|c|}{$\eta_{\mathrm{H}^{+}}$} & \multicolumn{4}{|c|}{$\eta_{\mathrm{g}}$} & \multirow{3}{*}{$\eta_{\mathrm{Cl}_{2}}$} & \multirow{3}{*}{$\eta_{\mathrm{O}_{2}}$} \\
\hline & & & \multicolumn{4}{|c|}{$C_{\mathrm{Cl}^{-}}^{\mathrm{S}}\left(\mathrm{kmol} / \mathrm{m}^{3}\right)$} & \multicolumn{4}{|c|}{$C_{\mathrm{Cl}}^{\mathrm{S}}\left(\mathrm{kmol} / \mathrm{m}^{3}\right)$} & & \\
\hline & & & 0.34 & 0.584 & 3.0 & 4.34 & 0.34 & 0.584 & 3.0 & 4.34 & & \\
\hline $\mathrm{K}-1$ & 0.34 & 93.1 & 18.0 & 14.2 & - & - & 111.1 & 107.3 & - & - & 86.2 & 13.8 \\
\hline $\mathrm{K}-2$ & 3.0 & 97.8 & - & - & 8.6 & 4.0 & - & - & 106.4 & 101.8 & 98.0 & 2.0 \\
\hline
\end{tabular}

$\eta_{\mathrm{g}}=\eta_{\mathrm{Ni}, \mathrm{Co}, \mathrm{Cu}}+\eta_{\mathrm{H}^{+}}$

$C_{\mathrm{Cl}^{-}}^{\mathrm{S}}: \mathrm{Cl}^{-}$-Konzentration nach Schluß der elektrolyse

$\eta_{\mathrm{Cl}_{2}}$, was darauf zurückzuführen sein dürfte, $\mathrm{da} ß$ ein Teil des entstehenden Sauerstoffs den Nickelfeinstein oxidiert. Nimmt man aufgrund des $\mathrm{H}^{+}$-Verhältnisses in $\mathrm{Abb} .4$ an, daß der Nickelfeinstein in den ersten und zweiten Oxidationsstufen durch die gesamte Chlor Menge und etwa $82 \%$ des entstehenden Sauerstoffs oxidiert wird und in der dritten Oxidationsstufe lediglich durch Chlor, dann beträgt für die anodische Oxidation von $\mathrm{Ni}, \mathrm{Co}$ und $\mathrm{Cu}$ die durchschnittliche Stromausbeute einen Wert von $93.0 \%$, der mit $\eta_{\mathrm{Ni}, \mathrm{Co}, \mathrm{Cu}}=93.1 \%$ in Tab. 4 gut übereinstimmt. Tatsächlich war die $\mathrm{O}_{2-}$ Entwicklung in den ersten und zweiten Oxydationsstufen gering und während der Elektrolyse war keine $\mathrm{Cl}_{2}$-Entwicklung wahrnehmbar.

Wie Tab. 4 zeigt, beträgt die gesamte Stromausbeute $\quad\left(\eta_{\mathrm{g}}=\eta_{\mathrm{Ni}, \mathrm{Co}, \mathrm{Cu}}+\eta_{\mathrm{H}^{+}}\right) \quad 111 \%$ bei $C_{\mathrm{NaCl}}=0.34 \mathrm{kmol} / \mathrm{m}^{3}$ und $106 \%$ bei $C_{\mathrm{NaCl}}=3.0 \mathrm{kmol} / \mathrm{m}^{3}$, wenn sich während der Elektrolyse die $\mathrm{Cl}^{-}$-Konzentration im Anolyten nicht ändert. In diesem Fall wurde die Stromausbeute für $\mathrm{H}^{+}$-Bildung, unter der Annahme, daß die $\mathrm{H}^{+}$-Ionen nach

$$
\mathrm{H}_{2} \mathrm{O}=2 \mathrm{H}^{+}+\frac{1}{2} \mathrm{O}_{2}+2 \mathrm{e}
$$

entstehen, aus den pH-Meßwerten mit Hilfe der Titrationskurven in Abb. 3 errechnet. Als Ursachen, daß $\eta_{\mathrm{g}} 100 \%$ übersteigt, kommen in Betracht

(1) eine Zunahme des aktivitätskoeffizienten der $\mathrm{H}^{+}$-Ionen im Anolyten durch elektrochemische $\mathrm{Cl}^{-}$-Ionenwanderung vom Kathodenraum in den Anodenraum und anodische Bildung von $\mathrm{Ni}^{2+}$ im Anodenraum. Das bedeutet eine Zunahme des $\mathrm{NiCl}_{2}$-Gehalts im Anolyten. Der Analysenwert der $\mathrm{Cl}^{-}$-Ionen im Anolyten am Schluß der Elektrolyse erreichtete $0.584 \mathrm{kmol} / \mathrm{m}^{3}$ bei $C_{\mathrm{NaCl}}=0.34 \mathrm{kmol} /$ $\mathrm{m}^{3}$ und $4.34 \mathrm{kmol} / \mathrm{m}^{3}$ bei $C_{\mathrm{NaCl}}=3.0 \mathrm{kmol} / \mathrm{m}^{3}$. Die entsprechenden Titrationskurven sind mit (1) und (2) in Abb. 3 wieder dargestellt. Der Aktivitätskoeffizient der $\mathrm{H}^{+}$-Ionen wird danach mit steingender $\mathrm{Cl}^{-}$-Konzentration von $0.34 \mathrm{kmol} / \mathrm{m}^{3}$ auf $0.584 \mathrm{kmol} / \mathrm{m}^{3}$ bzw. von $3.0 \mathrm{kmol} / \mathrm{m}^{3}$ auf $4.34 \mathrm{kmol} / \mathrm{m}^{3} 1.26$ - bzw. 2.20-mal größer. Unter diesen Bedingungen wird $\eta_{\mathrm{H}^{+}}$bei $C_{\mathrm{Cl}^{-}}^{\mathrm{s}}\left(\mathrm{Cl}^{-}\right.$-Konzentration nach Schluß der Elektrolyse) $=0.584 \mathrm{kmol} / \mathrm{m}^{3}$ bzw. $4.34 \mathrm{kmol} / \mathrm{m}^{3} 14.2 \%$ bzw. $4.0 \%$ und damit $\eta_{\mathrm{g}}$ $107.3 \%$ bzw. $101.8 \%$. $\eta_{\mathrm{g}}$ für $C_{\mathrm{NaCl}}=0.34$ $\mathrm{kmol} / \mathrm{m}^{3}$ ist damit immer noch zu hoch.

(2) Eine $\mathrm{H}^{+}$-Entstehung durch Oxidationsreaktionen des Nickelsulfids.

In diesem Fall kann auf die Bildungsreaktionen von Thioschwefelsäure-, Dithionsäure-, Polythionsäure, Schweflige Säure und Schwefelsäure-Ionen geschloßen werden. In Tab. 5 sind vergleichsweise die gesamte Stromausbeute $\left(\eta_{\mathrm{g}}\right)$ und das Verhältnis $\left(\varepsilon_{\mathrm{s}}\right)$ der tatsächlich gebildeten Elementarschwefelmenge zur theoretisch gebildeten Elementarschwefelmenge angegeben, die unter der Voraussetzung, daß eine dieser Reaktionen mit den Reaktionen von Gln. (3) und (11) gleichzeitig abläuft, berechnet wurden. Daraus ergibt sich, 
Tab. 5 Die gesamte Stromausbeute $\left(\eta_{\mathrm{g}}\right)$ und das Verhältnis $\left(\varepsilon_{\mathrm{s}}\right)$ der tatsächlich gebildeten Elementalschwefelmenge zur theoretisch gebildeten elementarschwefelmenge, wenn eine der Entstehungsreaktionen von Thioschwefelsäure-, Dithionsäure-; Polythionsäure-, schweflige Säureund Schwefelsäure-Ionen mit den Reaktionen von (3) and (11) nebeneinander erfolgt.

\begin{tabular}{|c|c|c|c|c|}
\hline \multirow[t]{2}{*}{ Nebenreajtionen } & \multicolumn{2}{|c|}{$\eta_{g}(\%)$} & \multicolumn{2}{|c|}{$\varepsilon_{\mathrm{s}}(\%)$} \\
\hline & $\begin{array}{c}\mathrm{K}-1 \\
C_{\mathrm{NaCl}}= \\
0.34 \mathrm{kmol} / \mathrm{m}^{3}\end{array}$ & $\begin{array}{c}C_{\mathrm{NaCl}}^{\mathrm{K}-2}= \\
3.0 \mathrm{kmol} / \mathrm{m}^{3}\end{array}$ & $\begin{array}{c}\mathrm{K}-1 \\
C_{\mathrm{NaCl}}= \\
0.34 \mathrm{kmol} / \mathrm{m}^{3}\end{array}$ & $\begin{array}{c}\mathrm{K}-2 \\
C_{\mathrm{NaCl}}= \\
3.0 \mathrm{kmol} / \mathrm{m}^{3}\end{array}$ \\
\hline $\mathrm{H}_{2} \mathrm{O}=2 \mathrm{H}^{+}+1 / 2 \mathrm{O}_{2}+2 \mathrm{e}$ & 107.3 & 101.8 & 90 & 103 \\
\hline $\mathrm{NiS}+3 / 4 \mathrm{H}_{2} \mathrm{O}=\mathrm{Ni}^{2+}+1 / 8 \mathrm{~S}_{8} \mathrm{O}_{6}^{2-}+3 / 2 \mathrm{H}^{+}+3.25 \mathrm{e}$ & 106.1 & 101.5 & 217 & 104 \\
\hline $\mathrm{NiS}+3 / 2 \mathrm{H}_{2} \mathrm{O}=\mathrm{Ni}^{2+}+1 / 2 \mathrm{~S}_{2} \mathrm{O}_{3}^{2-}+3 \mathrm{H}^{+}+4 \mathrm{e}$ & 104.7 & 101.1 & 127 & 108 \\
\hline $\mathrm{NiS}+3 / 2 \mathrm{H}_{2} \mathrm{O}=\mathrm{Ni}^{2+}+1 / 4 \mathrm{~S}_{4} \mathrm{O}_{6}^{2-}+3 \mathrm{H}^{+}+4.5 \mathrm{e}$ & 106.1 & 101.5 & 127 & 108 \\
\hline $\mathrm{NiS}+3 \mathrm{H}_{2} \mathrm{O}=\mathrm{Ni}^{2+}+1 / 2 \mathrm{~S}_{2} \mathrm{O}_{6}^{2-}+6 \mathrm{H}^{+}+7 \mathrm{e}$ & 106.1 & 101.5 & 106 & 106 \\
\hline $\mathrm{NiS}+3 \mathrm{H}_{2} \mathrm{O}=\mathrm{Ni}^{2+}+\mathrm{SO}_{3}^{2-}+6 \mathrm{H}^{+}+6 \mathrm{e}$ & 104.7 & 101.1 & 106 & 106 \\
\hline $\mathrm{NiS}+3 \mathrm{H}_{2} \mathrm{O}=\mathrm{Ni}^{2+}+\mathrm{HSO}_{3}^{-}+5 \mathrm{H}^{+}+6 \mathrm{e}$ & 105.8 & 101.4 & 109 & 106 \\
\hline $\mathrm{NiS}+4 \mathrm{H}_{2} \mathrm{O}=\mathrm{Ni}^{2+}+\mathrm{SO}_{4}^{2-}+8 \mathrm{H}^{+}+8 \mathrm{e}$ & 105.4 & 101.3 & 101 & 105 \\
\hline $\mathrm{NiS}+4 \mathrm{H}_{2} \mathrm{O}=\mathrm{Ni}^{2+}+\mathrm{HSO}_{4}^{-}+7 \mathrm{H}^{+}+8 \mathrm{e}$ & 106.3 & 101.5 & 103 & 105 \\
\hline
\end{tabular}

daß bei $C_{\mathrm{NaCl}}=3.0 \mathrm{kmol} / \mathrm{m}^{3}$ die Oxidation von NiS unter vollständiger Elementarschwefelbildung abläuft, während bei $C_{\mathrm{NaCl}}=0.34$ $\mathrm{kmol} / \mathrm{m}^{3}$ eine geringe Schwefelsäureionenbildungsreaktion sich überlagert. Aus diesen Ergebnissen lassen sich für die Änderung des $\mathrm{H}^{+}$-Verhältnisses in Abb. 4 folgende Schlüsse ziehen:

Für $C_{\mathrm{NaCl}}=0.34 \mathrm{kmol} / \mathrm{m}^{3}$ Lösungen findet in der ersten und zweiten Oxidationsstufe die $\mathrm{H}^{+}$-Entstehung durch eine Reaktion nach Gl. (11) statt. Die Zunahme der $\mathrm{H}^{+}$-Ionenkonzentration ist aber tatsächlich gering, da ca. $82 \%$ der entstehenden $\mathrm{H}^{+}$-Ionen durch die Oxidation des Nickelfeinsteins $\left(\mathrm{Ni}^{0}+2 \mathrm{H}^{+}+1\right)$ $2 \mathrm{O}_{2}=\mathrm{Ni}^{2+}+\mathrm{H}_{2} \mathrm{O}, \mathrm{Ni}_{3} \mathrm{~S}_{2}+2 \mathrm{H}^{+}+1 / 2 \mathrm{O}_{2}=\mathrm{Ni}^{2+}$ $+2 \mathrm{NiS}+\mathrm{H}_{2} \mathrm{O}$ ) verbraucht werden. In der dritten Oxidationsstufe ist der nach Gl. (11) entstehende Sauerstoff nicht in der Lage, den Nickelfeinstein (NiS) zu oxidieren, und dürfte wenn auch in geringen Maße die Reaktion der Schwefelsäureionenbildung die Reaktionen von Gln. (3) und (11) begleiten. Außerdem ist die Änderung des Aktivitätskoeffizienten der $\mathrm{H}^{+}$-Ionen mit steigender $\mathrm{Cl}^{-}$-Konzentration während der Elektrolyse gering. Bei konstantem Zellenstrom wird daher das $\mathrm{H}^{+}$-Verhältnis hauptsächlich durch die Geschwindigkeit der Reaktion nach Gl. (11) bestimmt und ändert sich in der dritten Oxidationsstufe rasch und geradlinig. Im Falle von $C_{\mathrm{NaCl}}=3.0 \mathrm{kmol} / \mathrm{m}^{3}$ hängt das $\mathrm{H}^{+}$-Verhältnis nur von der Geschwindigkeit der Reaktion nach Gl. (11) ab. Da, jedoch, die $\mathrm{Cl}^{-}$-Konzentration im Anolyten während der Elektrolyse allmählich ansteigt und damit der Aktivitätskoeffizient der $\mathrm{H}^{+}$Ionen zunimmt, scheint die $\mathrm{H}^{+}$-Entstehungsgeschwindigkeit (die Änderung des $\mathrm{H}^{+}$Verhältnisses) scheinbar beschleunigt $\mathrm{zu}$ werden.

\section{Konvexe Zelle}

In Abb. 5 ist der zeitlicher Verlauf des Suspensionspotentials und des pH-Werts des Anolyten für die Anfangssuspensionskonzentration des Nickelfeinsteins von $200 \mathrm{~kg} / \mathrm{m}^{3}$ (Vers.-Nr.: G-3) und $133 \mathrm{~kg} / \mathrm{m}^{3}$ (Vers.-Nr. G-

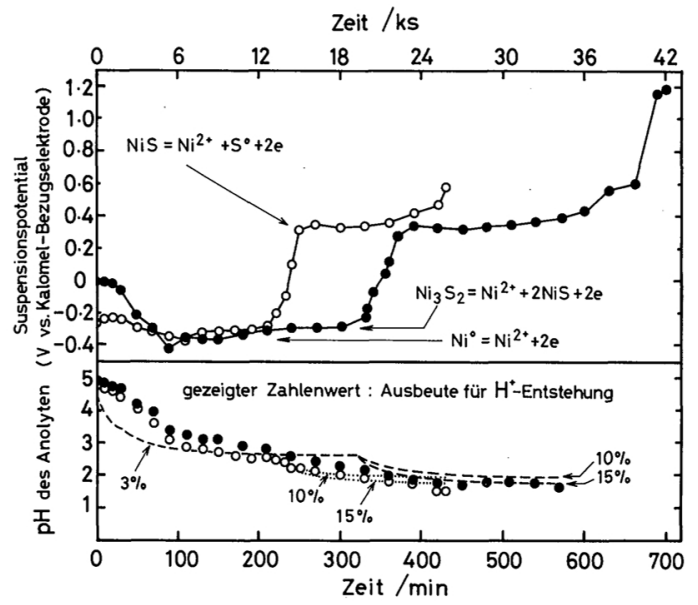

Abb. 5 Zeitlicher Verlauf des Suspensionspotentials und des $\mathrm{pH}$-Werts von Anolyten für NaCl-Gehalt von 3.0 $\mathrm{kmol} / \mathrm{m}^{3}$ bei der konvexen Zelle.

- G-3, O G-4 
4) bei einem $\mathrm{NaCl}-G e h a l t$ von $3.0 \mathrm{kmol} / \mathrm{m}^{3}$ und einem Zellenstrom von $20 \mathrm{~A}$ dargestellt. Die Ergebnisse der anodischen Oxidation des Nickelfeinsteins bestätigen die Versuchswerte bei den Kleinversuchen und die Ausbeute für die Elementarschwefelbildung erreicht 101\% bzw. 106\% für Vers.-Nr.: G-3 bzw. G-4. Bei diesen Versuchen fließt der Elektrolyt aus dem Kathodenraum durch die Trennwand in den Anodenraum, so daß die $\mathrm{Cl}^{-}$-Konzentration im Anolyten unverändert bleibt. Unter dieser Voraussetzung läßt sich die zeitliche Änderung der $\mathrm{H}^{+}$-Ionenkonzentration des Anolyten aus dem Stoffausgleich der $\mathrm{H}^{+}$-Ionen im Anodenraum nach Gl. (12),

$$
V \frac{\mathrm{d} C_{\mathrm{H}^{+}}}{\mathrm{d} t}=v C_{\mathrm{H}^{+}}^{\text {ein }}+n_{\mathrm{H}^{+}}-v C_{\mathrm{H}^{+}}^{\text {aus }},
$$

theoretisch ermitteln. Bei der Ableitung dieser Gleichung wird eine vollständige Mischung des Anolyten angenommen. Hierbei bedeutet $C_{\mathrm{H}^{+}}$ die $\mathrm{H}^{+}$-Ionenkonzentration des anolyten in $\mathrm{kmol} / \mathrm{m}^{3}, C_{\mathrm{H}^{+}}^{\mathrm{ein}}$ die $\mathrm{H}^{+}$-Ionenkonzentration des in den Anodenraum einfließenden Elektrolyten in $\mathrm{kmol} / \mathrm{m}^{3}, C_{\mathrm{H}^{+}}^{\text {aus }}$ die $\mathrm{H}^{+}$-Ionenkonzentration des aus dem Andenraum ausfließenden anolyten (deshalb $C_{\mathrm{H}^{+}}^{\text {aus }}=C_{\mathrm{H}^{+}}$) in $\mathrm{kmol} / \mathrm{m}^{3}, n_{\mathrm{H}^{+}}$die $\mathrm{H}^{+}$Entstehungsgeschwindigkeit im Anodenraum in $\mathrm{kmol} / \mathrm{s}, V$ das Volumen des Anodenraums in $\mathrm{m}^{3}$ und $v$ die Durchflußgeschwindigkeit des Elektrolyten in $\mathrm{m}^{3} / \mathrm{s}$. Unter der Anfangsbedingung von $C_{\mathrm{H}^{+}}=C_{\mathrm{H}^{+}}^{0}$ für $t=0$ ist Gl. (12) lösbar.

$$
C_{\mathrm{H}^{+}}=\left(C_{\mathrm{H}^{+}}^{\text {ein }}+\frac{n_{\mathrm{H}^{+}}}{v}\right)-\frac{\left(C_{\mathrm{H}^{+}}^{\text {ein }}-C_{\mathrm{H}^{+}}^{0}\right)+\frac{n_{\mathrm{H}^{+}}}{v}}{\exp \left(\frac{v}{V} t\right)} .
$$

Aus Gl. (13) läßt sich mit Hilfe der Titrationskurven in Abb. 3 die zeitliche $\mathrm{pH}$ - Änderung mit der gestrichelten Linie für Vers.-Nr.: G-3 und mit der gepunkteten Linie für Vers.-Nr.: G-4 in Abb. 5 wiedergegeben, wobei als anodische Stromausbeute der $\mathrm{H}^{+}$-Entstehung in der ersten und zweiten Oxidationsstufen bzw. in der dritten Oxidationsstufe 3\% bzw. 10 und $15 \%$ angenommen worden ist. Am Beginn der ersten und zweiten Oxidationsstufe liegen die pH-Meßwerte für Vers.-Nr.: G-4 oberhalb der theortischen Linie und erst am Ende dieser Stufen stimmen die beiden miteinander überein, während bei Vers.-Nr.: G-3 sich die Lage umgekehrt. Daraus ist zu ersehen, daß die Stromausbeute der $\mathrm{H}^{+}$-Entstehung in der ersten und zweiten Oxidationsstufen nicht konstant ist, sondern von kleinen Anfangswerten zu Versuchsbeginn allmählich zunimmt und im Durchschnitt etwa $3 \%$ erreicht. in der dritten Oxidationsstufe stimmen die theoretischen Werte für die Stromausbeute der $\mathrm{H}^{+}$-Bildung (10-15\%) mit den $\mathrm{pH}-\mathrm{Me} ß w e r t e n$ gut überein. Das deutet darauf hin, daß die Stromausbeute der $\mathrm{Cl}_{2}$-Entwicklung nach Gl. (4) höchstens ca. $90 \%$ erreicht.

Ein Vergleich der Stromausbeutewerte $\left(\eta_{\mathrm{Ni}, \mathrm{Co}, \mathrm{Cu}}\right)$ der anodischen Oxidation von $\mathrm{Ni}, \mathrm{Co}$ and $\mathrm{Cu}$ für NaCl-Gehalte von $0.34 \mathrm{kmol} / \mathrm{m}^{3(1)}$ und $3.0 \mathrm{kmol} / \mathrm{m}^{3}$ in Tab. 6 zeigt, daß die Werte bei $3.0 \mathrm{kmol} / \mathrm{m}^{3}$ deutlich größer sind als bei $0.34 \mathrm{kmol} / \mathrm{m}^{3}$. Die niedrige Stromausbeute von $87.3 \%$ für Vers.-Nr.: G-3 ist darauf zurückzuführen, daß die Elektrolyse bei einem höheren Suspensionspotential als dem der dritten Oxidationsstufe fortgesetzt wurde. Bei normalen Bedingungen wie z. B. bei Vers.-Nr.: G4 beträgt die Stromasubeute $95 \%$. Dieser Wert entspricht der Stromausbeute für eine $\mathrm{Cl}_{2}$-Entwicklung von $93 \%$, die auch mit dem aus der zeitlichen $\mathrm{pH}$-Änderung abgeschätzten Wert, ca. $90 \%$, übereinstimmt. Somit ist die Stromausbeute der $\mathrm{Cl}_{2}$-Entwicklung bei der konvexen Zelle neidriger als bei der Kleinversuchszelle und bei der elektrolyse des Elektrolyten ohne Nickelfeinsteinsuspensionen. Diese Abweichungen dürften auf den unterschiedlichen geometrischen Bau der Ableitanode zurückzuführen sein. Bei der Kleinversuchszelle besteht die Ableitanode aus einem spiralförmigen Platin-Draht und bei der konvexen Zelle aus einem um ein Titan-Netz gewickelten Platin-Draht. Im letzteren Falle könnte daher der Transport von $\mathrm{Cl}^{-}$-Ionen in die Spalte zwischen dem Titan-Netz und dem Platin-Draht ungenügend sein und damit statt der Entladung der $\mathrm{Cl}^{-}$-Ionen eine Wasserzersetzung erfolgen. Zur Verbesserung der Stromausbeute für die anodische Oxidation von $\mathrm{Ni}, \mathrm{Co}$ and $\mathrm{Cu}$, insbesondere in der dritten $\mathrm{Ox}$ - 
Tab. 6 Einfluß des $\mathrm{NaCl}-G e h a l t s$ im Anolyten auf die Stromausbeute $\left(\eta_{\mathrm{Ni}, \mathrm{Co}, \mathrm{Cu}}\right)$ für die anodische Oxidation von $\mathrm{Ni}, \mathrm{Co}$ und $\mathrm{Cu}$ und die nach Schluß der Elektrolyse im Anodernraum bleibende Rückstandsmenge bei der konvexen Zelle.

\begin{tabular}{cccccc}
\hline \hline Versuch-Nr. & $\begin{array}{c}\text { Zellenstrom } \\
\text { (A) }\end{array}$ & $\begin{array}{c}\text { NaCl-Gehalt } \\
\left(\mathrm{kmol} / \mathrm{m}^{3}\right)\end{array}$ & $\begin{array}{c}\text { pH-Wert des } \\
\text { Anolyten }\end{array}$ & $\begin{array}{c}\text { Konzentration } \\
\text { des Rückstands } \\
\left(\mathrm{kg} / \mathrm{m}^{3}\right)\end{array}$ & $\begin{array}{c}\eta_{\mathrm{Ni}, \mathrm{Co}, \mathrm{Cu}} \\
(\%)\end{array}$ \\
\hline G-1 & 20 & 0.34 & 1.0 & 29 & 83.0 \\
G-2 & 30 & 0.34 & $5.0^{*}$ & 28 & 83.3 \\
G-3 & 20 & 3.0 & $4.9^{*}$ & 6 & 87.3 \\
G-4 & 20 & 3.0 & $4.8^{*}$ & 9 & 95.5 \\
\hline \hline
\end{tabular}

*: Anfangswert

idationsstufe ist daher neben einer Erhöhung des NaCl-Gehaltes im Anolyten auch eine Optimierung der Bauart der Ableitanode von großer Bedeutung.

Aus den in Tab. 6 angegebenen Menge des Rückstands im Anodenraum nach Schluß der Elektrolyse ist $\mathrm{zu}$ ersehen, daß bei $\mathrm{NaCl}$ Gehalten von $0.34 \mathrm{kmol} / \mathrm{m}^{3}$ viel höhere Nickelfeinstein-Restgehalte als bei $3.0 \mathrm{kmol} / \mathrm{m}^{3}$ anfallen, was vermutlich dadurch verursacht wird, daß das entstehende Sauerstoffgas die Reaktion zwischen dem Nickelfeinstein und $\mathrm{Cl}_{2}$ (aq) behindert.

Über den Mechanismus und Ablauf der anodische Oxidation von $\mathrm{Ni}_{3} \mathrm{~S}_{2}, \beta$-NiS und Nickelstein wird im Schrifttum berichtet, daß sich diese Sulfide bei in Lösung gehenden Nickelionen über eine Reihe von Zwischenphasen u. zw. von $\mathrm{Ni}_{1,5} \mathrm{~S}_{2} \rightarrow \mathrm{Ni}_{1,2} \mathrm{~S}_{2} \rightarrow \mathrm{NiS}_{2} \rightarrow$ $\mathrm{Ni}^{2+}+\mathrm{S}^{0}$ zersetzen $^{(12)(13)}$. Bei den vorliegenden Untersuchungen konnten, jedoch, diese Zwischenphasen nicht festgestellt werden.

Den anodische Umsetzungsmechanismus betreffend ist sowohl eine unmittelbare Oxidation durch direkte Berühung der Metallsulfidpartikeln mit der Ableitanode als auch eine mittelbare Oxidation durch Oxidationsmittel, die an der Anode gebildet werden, möglich. Da die Stromausbeute für die anodische Oxidation von $\mathrm{Ni}, \mathrm{Co}$ und $\mathrm{Cu}$, jedoch, maßgeblich vom $\mathrm{NaCl}-G e h a l t$ im Anolyten abhängt, dürfte die Stoffumsetzung mit Oxidationsmitteln den Elektrolyseablauf bestimmen. In diesem Fall wird als Oxidationsmittel $\mathrm{HClO}(\mathrm{aq})$ im relativ hohen $\mathrm{pH}$-Bereich und $\mathrm{Cl}_{2}$ (aq) im relativ niedrigen $\mathrm{pH}$-Bereich dominieren. Dieser Umsetzungsmechanismus erlaubt, die anodische Auflösung des Nickelfeinsteins aus dem
Anodenraum in einen mit der Elektrolysezelle verbundenen Laugungsbehälter zu verlagern. Aufgrund dieser Überlegung durchgeführte elektrolytische Laugungsversuche mit Nickelfeinstein zeigen gute Ergebnisse, die in einem weiteren Bericht veröffentlicht werden.

\section{Zusammenfassung}

Untersuchungen über die anodische Oxidation von Nickelfeinsteinsuspensionen in konzentrierten $\mathrm{NaCl}-L$ ösungen wurden mit Hilfe einer Kleinzelle und einer konvexen Zelle durchgeführt, wobei auch die Eigenschaften des NaCl-haltigen Elektrolyten ermittelt wurden.

Gleichgewichtsberechnungen des Systems $\mathrm{Cl}_{2}$ (g)- $\mathrm{Cl}_{2}$ (aq)-HClO(aq)-ClO ${ }^{-}$in NaCl-haltigen Lösungen zeigen, daß bei der Nickelfeinstein-Suspensionselektrolyse als Oxidationsmittel im niedrigen $\mathrm{pH}$-Bereich $\mathrm{Cl}_{2}(\mathrm{aq})$ und im relativ hohen, aber $<5 \mathrm{pH}$-Bereich $\mathrm{HClO}(\mathrm{aq})$ wirksam sind. Die Nickelfeinstein-Suspensionen werden anodisch in drei Stufen, $\mathrm{Ni}^{0}$ $=\mathrm{Ni}^{2+}+2 \mathrm{e}, \mathrm{Ni}_{3} \mathrm{~S}_{2}=\mathrm{Ni}^{2+}+2 \mathrm{NiS}+2 \mathrm{e}$ und $\mathrm{NiS}$ $=\mathrm{Ni}^{2+}+\mathrm{S}^{0}+2 \mathrm{e}$ durch die anodisch gebildeten Chlor oder Chlorverbindungen sowie Sauerstoff oxidiert. Das Zwischenprodukt, NiS, kann jedoch durch Sauerstoff nicht oxidiert werden. In der Kleinzelle erreichte die anodische Stromausbeute für die Oxidation von $\mathrm{Ni}, \mathrm{Co}$ und $\mathrm{Cu}$ beim $C_{\mathrm{NaCl}}=0.34 \mathrm{kmol} / \mathrm{m}^{3}$ bzw. $3.0 \mathrm{kmol} / \mathrm{m}^{3}$ 93\% bzw. 98\%. Diese Werte hängen maßgeblich von der anodisch gebildeten Gaszusammensetzung $\left(\mathrm{Cl}_{2}: \mathrm{O}_{2}\right)$ ab. In der konvexen Zelle waren niedrigere Stromausbeuten von $83 \%$ bzw. $96 \%$ zu verzeichnen, die auf eine geringere $\mathrm{Cl}_{2}$-Entwicklung infolge ungünstiger Bauart der 
Ableitanode zurückzuführen sind.

\section{Danksworte}

Die Durchführung dieser Arbeit wurde für Prof. Dr.-Ing. Ch. Yamauchi sowohl von der Alexander von Humboldt-Stiftung als auch vom Ministry of Education of Japan durch Grant-in-Aid Scientific Research unterstützt. Dafür sei auch an dieser Stelle gedankt.

\section{SCHRIFTTUM}

(1) Ch. Yamauchi, H. Kametani und R. Kammel: Erzmetall, 33 (1980), 586.

(2) Ch. Yamauchi, H. Kametani und R. Kammel: Metall, 35 (1981), 401.

(3) M. Takahashi: Soda to Enso, 29 (1978), 379.

(4) M. Takahashi und N. Masuko: Kogyo Denkai no
Kagaku, Agune (1st Ed.), Tokio, (1979).

(5) F. Förster und E. Müller: Z. Elektrochem., 9 (1903), 171.

(6) M. W. Lister: Can. J. Chem., 30 (1952), 879, 31 (1956), 479.

(7) I. Taniguchi und T. Sekine: Denki Kagaku, 43 (1975), 715.

(8) H. Imagawa: Denki Kagaku, 21 (1953), 520.

(9) M. M. Jaksic: J. Electrochem. Soc., 121 (1974), 70.

(10) B. V. Tilak, K. Viswanathan und C. G. Rader: ibid., 128 (1981), 1228.

(11) H. S. Harned und B. B. Owen: The Physical Chemistry of Electrolytic Solutions, Reinhold, (1958), S. 727.

(12) D. C. Price und W. G. Davenport: J. Appl. Electrochem., 12 (1982), 281.

(13) T. Kato und T. Oki: J. Japan Inst. Metals, 37 (1973), 1338 (in Japanese). 